\title{
Hypopharyngeal Cancer pT2 TNM Finding v8
}

National Cancer Institute

\section{Source}

National Cancer Institute. Hypopharyngeal Cancer pT 2 TNM Finding v8. NCI Thesaurus. Code C132930.

Hypopharyngeal cancer with tumor invading more than one subsite of hypopharynx or an adjacent site, or measuring more than $2 \mathrm{~cm}$ but not more than $4 \mathrm{~cm}$ in greatest dimension without fixation of hemilarynx. (from AJCC 8th Ed.) 